\title{
New injection and acceleration scheme of positrons in the laser-plasma bubble regime
}

\author{
Z. Y. Xu, ${ }^{1}$ C. F. Xiao, ${ }^{1}$ H. Y. Lu๑ ${ }^{1,2,3, *}$ R. H. Hu ${ }^{1, \dagger}$ J. Q. Yu, ${ }^{1, \$}$ Z. Gong $\odot,{ }^{1}$ Y. R. Shou, ${ }^{1}$ \\ J. X. Liu, ${ }^{1}$ C. Z. Xie $\odot,{ }^{1}$ S. Y. Chen, ${ }^{1}$ H. G. Lu, ${ }^{1}$ T. Q. Xu, ${ }^{1}$ R. X. Li, ${ }^{4}$ N. HafZ $\odot,{ }^{5}$ \\ S. Li, ${ }^{5}$ Z. Najmudin, ${ }^{6}$ P. P. Rajeev, ${ }^{7}$ D. Neely, ${ }^{7}$ and X. Q. Yan ${ }^{1,3}$ \\ ${ }^{1}$ Key Laboratory of HEDP of the Ministry of Education, CAPT, and State Key Laboratory of Nuclear \\ Physics and Technology, CAPT, Peking University, Beijing 100871, China \\ ${ }^{2}$ Center for Advanced Material Diagnostic Technology, College of Engineering Physics, \\ Shenzhen Technology University, Shenzhen 518118, China \\ ${ }^{3}$ Collaborative Innovation Center of Extreme Optics, Shanxi University, Taiyuan, Shanxi 030006, China \\ ${ }^{4}$ State Key Laboratory of High Field Laser Physics, Shanghai Institute of Optics and Fine Mechanics, \\ Chinese Academy of Sciences, Shanghai 201800, China \\ ${ }^{5}$ ELI-HU Non-Profit Ltd., Dugonics tr 13, Szeged 6720, Hungary \\ ${ }^{6}$ Blackett Laboratory, Imperial College, London SW7 2AZ, United Kingdom \\ ${ }^{7}$ Central Laser Facility, Rutherford Appleton Laboratory, Didcot, OX11 OQX, United Kingdom
}

(Received 8 December 2019; revised 10 May 2020; accepted 12 August 2020; published 1 September 2020)

A novel approach for positron injection and acceleration in the laser driven plasma wakefield is proposed. One ring-shaped beam and one coaxially propagating Gaussian beam drive wakefields in a preformed plasma volume filled with both electrons and positrons. The laser's ponderomotive force as well as the charge separation force in the front bucket of the first bubble are utilized to provide the transverse momenta of injected positrons and those positrons can be trapped by the focusing field and then accelerated by the wakefield. Theoretical analysis of the process is presented and verified by particle-in-cell simulations. The simulations show that relatively high-charge, quasimonoenergetic positron beams can be obtained.

DOI: 10.1103/PhysRevAccelBeams.23.091301

\section{INTRODUCTION}

Intense relativistic positron beams are crucial for pair production in the field of fundamental physics [1] and violent high-energy astrophysical phenomena [2]. A stable method to generate intense, monoenergetic and fully tunable positron beams enables experimental study of gamma-ray bursts and black holes $[3,4]$. Bremsstrahlungbased high-energy positrons are usually produced in linear accelerators (LINACs) and synchrotron facilities via propagating the relativistic electron beams through thick, high-Z targets. However, positron beams generated through this

\footnotetext{
*Corresponding author.

luhaiyang@sztu.edu.cn

Present address: School of Physics and Technology, Sichuan University.

ronghaohu@scu.edu.cn.

Present address: School of physics and Electronics, Hunan University.

jinqing.yu@hnu.edu.cn

Published by the American Physical Society under the terms of the Creative Commons Attribution 4.0 International license. Further distribution of this work must maintain attribution to the author(s) and the published article's title, journal citation, and DOI.
}

method have broadband energy spectra and large transverse divergence, which restrict their applications.

Since the concept of laser wakefield accelerator (LWFA) was first proposed in 1979 [5], many works have been done on electron acceleration using ultrafast laser systems [6-19]. Great progress has been made in producing high-quality electron beams [6-8,10-12] as well as in boosting the electron energy [12-14,19-22]. Recently, the generation of sub-hundred $\mathrm{MeV}$ positrons has been experimentally demonstrated by hitting these electron beams on high-Z solid targets [23]. However, the resulting positron beams are limited both in yield and energy. Hybrid schemes have also been proposed and conducted to generate low energy $(E<20 \mathrm{MeV})$ and broad divergence $(\sim 1 \mathrm{rad})$ positron jets with high positron yields (up to $10^{11}$ per shot) [24-26]. Positrons with high energy and small divergence can principally be achieved through laser-plasma acceleration [27-31]. However, a scheme for positron injection into these accelerators has yet been absent.

Ultrashort and intense ring-shaped laser propagating in plasma can excite donut-shaped bubbles, which are thought to be suitable structures for positron acceleration [27-31]. One typical ring-shaped laser is Laguerre-Gaussian pulse whose azimuthal and radial indexes are 1 and 0 , 
respectively. The difficulty of positron acceleration in the bubble regime [9] is that positrons, due to their positive charge, are easily expelled away from the bubble in the transverse direction $[32,33]$.

\section{METHOD OF INJECTION}

In the scheme, a novel proposal is presented for positron injection and acceleration, which we tentatively name "ballistic injection," as shown in Fig. 1. A ring-shaped laser beam and a coaxially propagating Gaussian laser beam are employed to create donut and center bubbles in the plasma, respectively. In the scheme, the positrons in the bubble can experience the process of scattering, trapping and acceleration before they are emitted or decelerated. In the beginning, the positrons are repelled by the electromagnetic force from the laser tail $[32,33]$ like ions scattered by nuclei. The positrons will be injected into the bubbles if the transverse momentum is high enough to let the positrons penetrate the bubble and stay inside. The transverse motion of the positrons is restricted by the focusing electromagnetic field inside the bubble. The longitudinal electric field can accelerate these positrons to relativistic velocities if they stay long enough inside the bubble.

In this scheme, two laser beams that have the same temporal profile but with a time delay $\tau$ are employed. Their normalized intensities are $a^{G}(t, r)=a_{0}^{G} \exp \left(-\frac{r^{2}}{r_{G}^{2}}-\frac{(t-\tau)^{2}}{t_{0}^{2}}\right)$

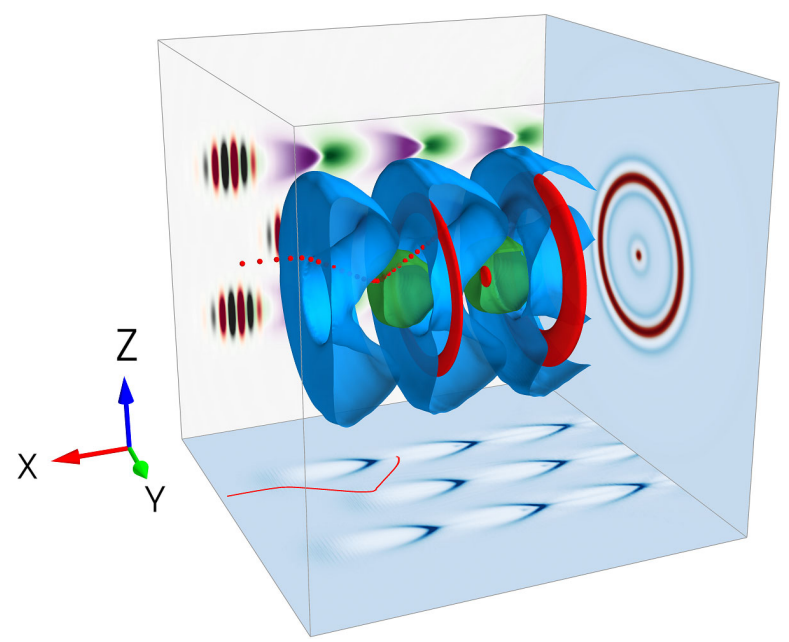

FIG. 1. The concept of the positron ballistic injection scheme. The blue and green colors are contour surfaces of electron densities of donut and center bubbles, respectively. The red color represents injected positrons. The $x-y$ and $x-z$ planes are transverse slices of the density distribution and the longitudinal electric field $E_{x}$. The red curve in the $x-y$ plane is the trajectory of an injected positron (corresponding to the projection of red balls in the 3D model). The leading oscillating colors (amber and grey) denote the laser beams in the $x-z$ plane. The $y-z$ plane is the projection of electron density (blue) and injected positron density (red). for the Gaussian beam and $a^{R}(t, r)=a_{0}^{R} \exp \left(-\frac{\left(r-r_{0}\right)^{2}}{r_{R}^{2}}-\frac{t^{2}}{t_{0}^{2}}\right)$ for the ring-shaped beam, where $r$ is the distance to laser propagation axis and $t$ is time. $a_{0}^{G}$ and $a_{0}^{R}$ are the normalized peak intensities, and $r_{G}$ and $r_{R}$ are the beam sizes of the Gaussian beam and ring-shaped beam respectively. $t_{0}$ is the pulse duration of both laser beams. A positive $\tau$ means the ring-shaped beam is ahead of the center Gaussian beam. For easy understanding, time delay $\tau$ is normalized to $\lambda_{p} / c$, where $\lambda_{p}=\frac{2 \pi v_{g}}{\omega_{p}}$ is the plasma wavelength. Here, $c$ is the speed of light in vacuum, $\omega_{p}=\sqrt{\frac{4 \pi n_{e} e^{2}}{m_{e}}}$ is the plasma frequency, $m_{e}$ is the electron or positron mass, $e$ is the unit charge of electrons (negative) or positrons (positive), $n_{e}$ is the electron density, $v_{g}=c \sqrt{1-n_{e} / n_{c}}$ is the group velocity of light in plasma, and $n_{c}$ is the critical density.

The spatial profile of the ring-shaped beam can be characterized by inner radius $r_{\text {in }}$ and outer radius $r_{\text {out }}$, or ring radius $r_{0}=\left(r_{\text {out }}+r_{\text {in }}\right) / 2$ and ring width $r_{d}=\left(r_{\text {out }}-r_{\text {in }}\right) / 2$. It can excite a series of donut bubbles with a width of $r_{d}=2 \sqrt{a_{0}^{R}} c / \omega_{p}$ when the matching condition is met, as predicted by nonlinear theory for bubbles [9]. To clarify the dynamic processes for the scattering and injection, it is better to let the two laser beams interact with their surrounding plasma independently. Thus, the relation $2\left(\sqrt{a_{0}^{G}}+\sqrt{a_{0}^{R}}\right) c / \omega_{p}<r_{0}$ should be satisfied. In this case, the excited plasma wakefields by Gaussian and ring-shaped laser beams can be analyzed independently.

In this scheme, the plasma region is initially uniformly filled with electrons (density $n_{e}$ ), and externally provided positrons (density $n_{p}$ ) for simplicity. The positron density $n_{p}$, is assumed to be much lower than $n_{e}$, e.g., $n_{p}=0.01 n_{e}$, to guarantee there is no apparent affection to the plasma field. The contribution of positrons can thus be neglected when analyzing the shape and fields of the plasma bubbles.

It is key to realize the transfer of positrons between the center and the donut bubbles. The scattering process provides the positrons with transverse momenta, which makes them possible to move from the center bubble to the donut bubble or from the donut bubble to the center bubble for trapping. Otherwise, the positrons will be pushed away by the front bucket of the first bubble which is usually overlapped with the rear of the laser pulse.

For laser propagating along the $x$ axis, under the quasistatic approximation, assumes all variables depend on $\xi=x-v_{g} t$ instead of $x$ and $t$. In the moving frame of the bubble, the currents, densities, and bubble boundaries are then time independent.

The scattering process of positrons is the result of both laser ponderomotive force and charge separation force of the bubbles. The laser ponderomotive force takes the form of $F_{p}=-\frac{1}{2 m_{e} \gamma} \nabla\left(\overline{\left|\frac{e A}{c}\right|^{2}}\right)=-\frac{m_{e} c^{2}}{2 \gamma} \nabla\left(\overline{a_{0}^{2}}\right)$. 
The average laser ponderomotive force can be estimated to be $\overline{F_{p}}=\frac{m_{e} c^{2} a_{0}^{2}}{4 W_{0} \sqrt{1+a_{0}^{2} / 2}}$, where $W_{0}$ is the laser spot size.

The total charge separation force on a positron inside the bubble [34] can be written as $F_{c}=\frac{m_{e} \omega_{p}^{2}}{2} r$. The total charge separation force on a positron acts as a conservative repulsive force, pointing from the center of the bubble to the position of the positron, with a strength proportional to the distance between them. The average charge separation force is estimated to be $\overline{F_{c}}=\frac{m_{e} \omega_{p}^{2}}{8} \lambda_{p}$. Thus, the ratio of laser ponderomotive force over charge separation force is determined to be $\delta=\frac{\overline{F_{p}}}{\overline{F_{c}}}=\frac{2 c^{2} a_{0}^{2}}{\omega_{p}^{2} \lambda_{p} W_{0} \sqrt{1+a_{0}^{2} / 2}}$.

Given an example case of $a_{0} \sim 10^{0}, n_{e} \sim 10^{18} \mathrm{~cm}^{-3}$, $W_{0} \sim 10 \mu \mathrm{m}$, thus $\delta<0.1$. It means the charge separation force is the primary scattering factor even in the first bubble, where the laser ponderomotive force indirectly contributes to the scattering effect, as shown in Fig. 2. The theoretical calculation result is directly derived from the charge separation force and does not include the term of laser ponderomotive force. The laser peak intensity $\left(a_{0}=2\right)$ is only used to determine the radius of the bubble. The theoretical scattering angle cuts off beyond the bubble radius at $b=2 \sqrt{2}$. The simulation results include the contributions of both the charge separation force and the laser ponderomotive force. Stable bubbles can be formed with high enough laser intensities, and the laser ponderomotive force increases with the square of $a_{0}$. However, the scattering angles increase very slightly. This verifies that the dominant force is the charge separation force of the bubbles, and the scattering process is affected by the laser

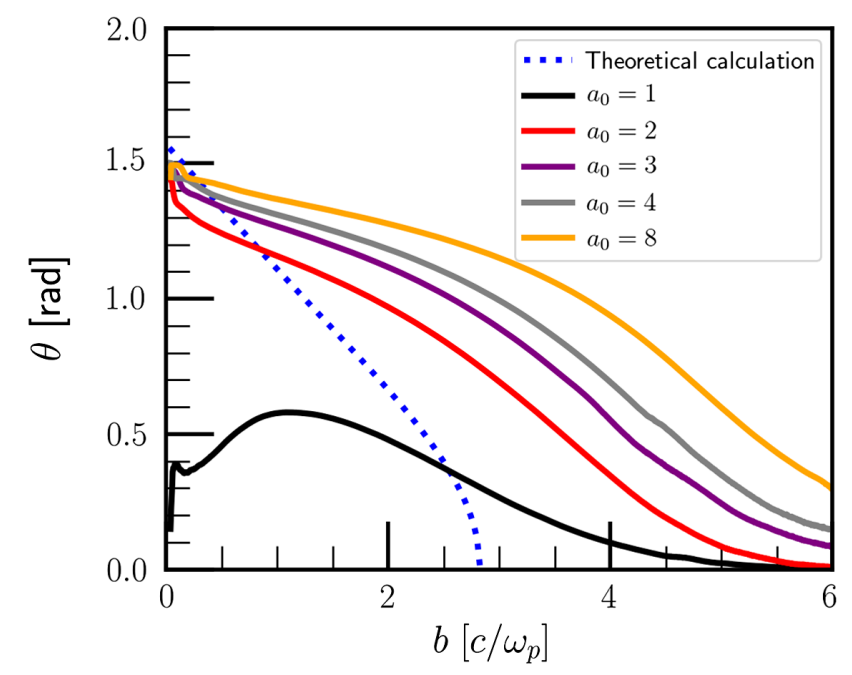

FIG. 2. The scattering angle $\theta$ of positrons by the first bubble as a function of different impact parameter $b$ (the positrons starting distance to laser axis) under different peak laser intensities. Theoretical calculation (blue dotted line) is estimated based on Eq. (1) with $a_{0}=2$, and solid lines are results from PIC simulations. indirectly through changing the shape of the bubbles. The scattering force in the second and the following bubbles comes solely from the charge separation force as the laser is absent due to its short pulse duration.

The form of the repulsive force keeps the trajectory of a positron in the same plane. For initially relativistic positrons, the relativistic factor can be treated as a constant, assuming that it does not change dramatically during the scattering process. $F_{c}$ can be solved as the Binet equation, giving the trajectory of positrons inside the bubble, which is a hyperbola. For a positron that enters the bubble at coordinate $\left(\xi_{0}, y_{0}\right)$ and has velocity $v_{0}$ along the $\xi$ axis, the solutions can be written as

$$
\begin{aligned}
& x=\frac{v_{0}}{\omega} \sinh \omega t+x_{0} \cosh \omega t \\
& y=y_{0} \cosh \omega t \\
& \omega=\sqrt{\frac{1}{2} \omega_{p} .}
\end{aligned}
$$

The scattering angle can be determined from the above equations.

In the accelerating stage, consider the spherical bubble excited by the Gaussian laser $a^{G}(t, r)=$ $a_{0}^{G} \exp \left(-\frac{r^{2}}{r_{G}^{2}}-\frac{(t-\tau)^{2}}{t_{0}^{2}}\right)$. For paraxial electrons and positrons, the one-dimensional fluid model is applied. The scalar potential $\phi(\xi)$ satisfies the Poisson-like equation $\frac{\partial^{2} \phi}{\partial \xi^{2}}=$ $k_{p}^{2} \gamma_{p}^{2}\left[v_{p}\left(1-\frac{1+\left(a^{G}\right)^{2}}{\gamma_{p}^{2}(1+\phi)^{2}}\right)^{-\frac{1}{2}}-1\right]$, where $\gamma_{p}=\left(1-v_{p}^{2} / c^{2}\right)^{-1 / 2}$ is the Lorentz factor corresponding to the phase velocity of the plasma wave, and $k_{p}=\omega_{p} / v_{p}$ is the wave vector. The Hamiltonian can be expressed as $H=\sqrt{1+p_{\|}^{2}+p_{\perp}^{2}} \pm \phi(\xi)$, where the minus and the plus signs are for electrons and positrons, respectively. $p_{\|}$and $p_{\perp}$ are the parallel and perpendicular momentum, respectively.

Under the canonical transformation $\left(x, p_{\|}\right) \rightarrow\left(\xi, p_{\|}\right)$, the Hamiltonian becomes $H=\sqrt{1+p_{\|}^{2}+p_{\perp}^{2}} \pm \phi(\xi)-v_{g} p_{\|}$. This gives several constants of motion. The first is the conservation of transverse canonical momentum, $p_{\perp} \pm a^{G}=$ const. For positrons initially at rest and far from a sufficiently short laser pulse, this is effectively $p_{\perp}=-a^{G}$.

Another constant of motion is the energy. The solution for longitudinal momentum for a positron with an initial energy $H_{0}$ is given as $p_{\|}=v_{p} \gamma_{p}\left(H_{0}-\phi\right) \pm$ $\gamma_{p} \sqrt{\gamma_{p}^{2}\left(H_{0}+\phi\right)^{2}-p_{\perp}^{2}-1}$. This solution gives the positron trajectory in $\left(\xi, p_{\|}\right)$phase space similar to Fig. 1 in Ref. [32]. The trapped orbits in the second and third bubbles show the potential of actual acceleration of positrons, as the transverse charge separation force acts as a focusing force. However, unlike electron self-injection, 
positrons initially at rest cannot be self-injected into these areas [32]. In this injection scheme, the positrons can gain enough transverse momenta, through scattering by other bubbles or the laser pulse, to penetrate the bubble and enter the trapped orbits transversely.

Wide transverse distribution of positrons will lead to the injection of positrons in different bubbles due to different scattering paths, which will result in multibunches. Even positrons with the same transverse position can experience the same acceleration field in the bubble, wider initial longitudinal distribution will lead to broadening of energy spectra of accelerated positrons in each bubble, similarly to the case of continuous injection of electrons in LWFA.

\section{SIMULATION RESULTS}

Particle-in-cell (PIC) simulations were conducted using code EPOCH [35] for detailed understanding of the injection and acceleration dynamics. The 2D simulation box corresponds to a physical volume of $120 \mu \mathrm{m} \times 160 \mu \mathrm{m}$, and is sampled by 20 cells per laser wavelength in the laser propagation direction and eight cells per wavelength in each transverse direction. The so-called "moving window" technique is used in simulations. It travels in the direction of the laser with a speed of $v_{g}$. Two macroelectrons, two macroprotons and 64 macropositrons are placed in each cell. Two coaxial laser beams propagate along the $x$ axis and are linearly polarized in the $x-y$ plane with $a_{0}^{G}=a_{0}^{R}=2$. The pulse duration is fixed to $20 \mathrm{fs}$ in full width at half maximum (FWHM) for both beams, and the wavelength is set to $0.8 \mu \mathrm{m}$. The center laser beam has a focused spot size of $10 \mu \mathrm{m}$ (FWHM). The ring-shaped beam has a ring radius $r_{0}=30 \mu \mathrm{m}$ and ring width $r_{d}=10 \mu \mathrm{m}$. The plasma region is placed $10 \mu \mathrm{m}$ away from the left boundary of the simulation box. The ambient plasma electron density is set to $n_{e}=3 \times 10^{18} \mathrm{~cm}^{-3}$, and the first $100 \mu \mathrm{m}$ of plasma region is filled with positrons. The positron density is assumed to be $n_{p}=1 \times 10^{16} \mathrm{~cm}^{-3}$ in the simulation, which is already experimentally demonstrated [24]. The initial temperature of positrons is assumed to be $2 \mathrm{MeV}$ which is considered to be higher enough to prevent annihilation with electrons before they can be accelerated. The Supplemental Material [36] describes the details of the simulations.

The focusing force on positrons in the front bucket of bubbles is the result of high-density electron sheath in the rear of the previous bubbles. Thus, the first donut or center bubble cannot hold positrons as lacking of this focusing force. In the simulations, almost all injected positrons are in the second and third center or donut bubbles, and positrons in the fourth and later bubbles can be neglected.

The ponderomotive force of driving laser and the charge separation force in the front bucket of the first bubble behave as defocusing forces on the positrons. The donut bubbles can capture and hold more positrons as their

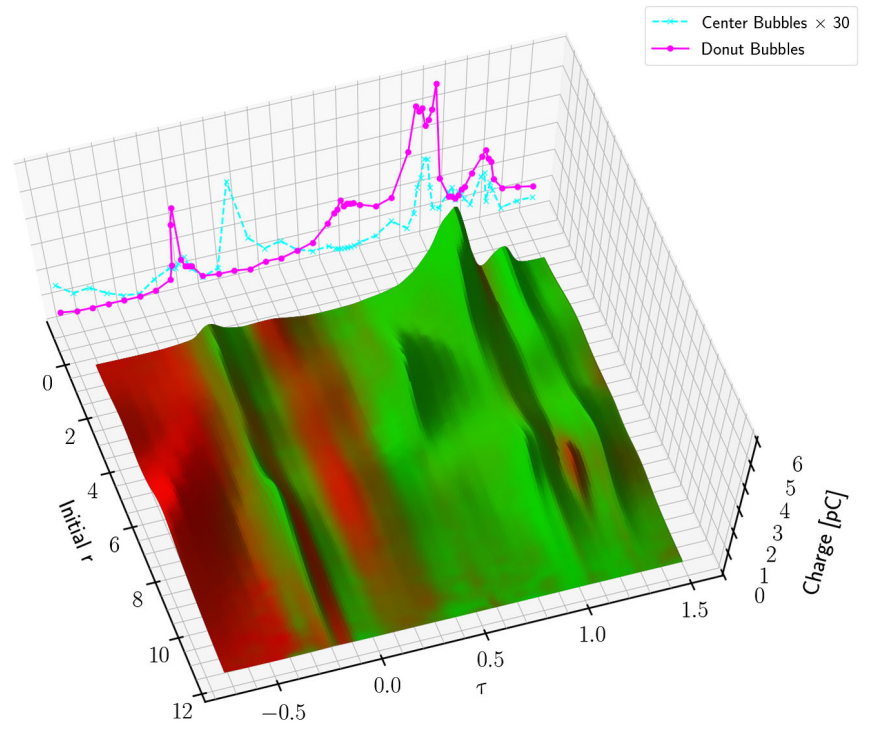

FIG. 3. The charge of injected positrons as a function of initial off-axis distance and delay in the front three consecutive donut and center bubbles. The value is the total charge, and the color denotes which kind of bubble is dominant, green (red) color means positron charges are mainly from center (donut) bubbles as an example.

volumes are much larger than the center bubbles. In the simulations, the pulse energy of the ring-shaped beam is 8 times higher than that of the Gaussian beam for the same normalized intensity. The volume of the excited donut bubble is roughly 12 times larger than the center bubble considering a 3D configuration. Thus, one donut bubble can hold more positrons than the center bubble by considering the 3D charge calculation shown in Fig. 3.

The initial transverse position $r$ plays a vital role in the injection of positrons as it will determine the scattering trajectory and the following injection position. Positrons initially distributed within radius from $r_{0} / 2$ to $r_{0}$ are mainly scattered by the donut bubble first, and those within the radius of $r_{0} / 2$ are scattered mainly by the center bubble first. It is noted that there are two peaks of the charge of injected positrons, with $r$ around 0 and $r_{d}$ (slightly smaller), for several different periodic delays $\tau$. The injection also terminates beyond $r_{d}$, because these positrons are scattered outward and leave the bubble region.

Different delay $\tau$ corresponds to different relative position of the center and donut bubbles. When one positron is scattered by one of the bubbles, the delay $\tau$ will determine the point where the positron enters the other bubbles. Thus, an appropriate delay $\tau$ can push a relatively large portion of scattered positrons into the transverse focusing field, where they are also in the accelerating phase, and results in the increase of the injection rates. For positive delays, positrons scattered by the first center bubble will go directly towards the second donut bubble. Only a very small fraction of positrons gains enough transverse momenta through one 


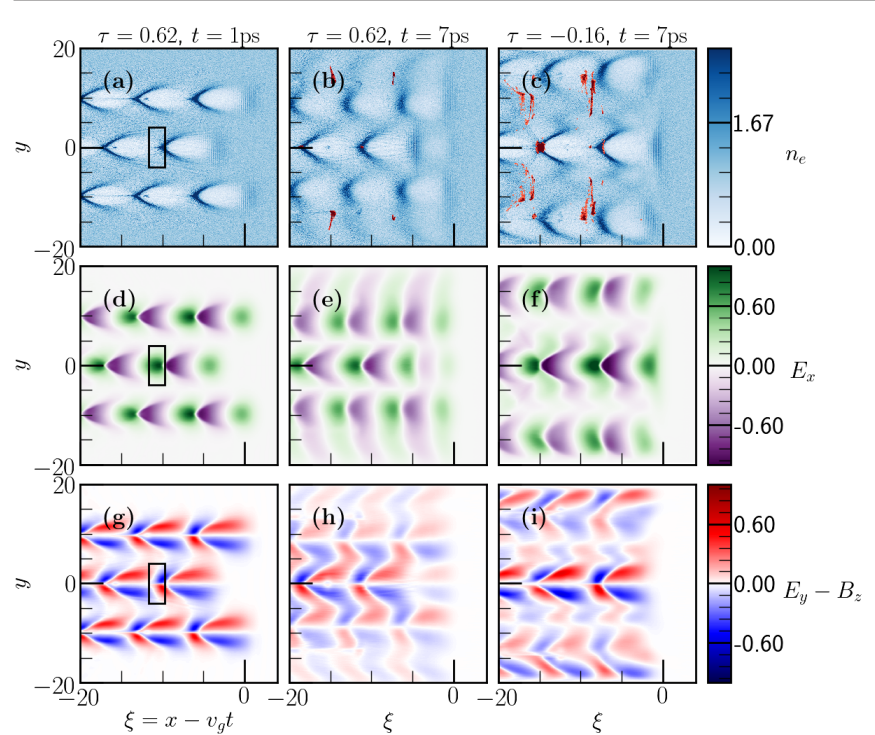

FIG. 4. Simulation results of (a)-(c): electron density distribution $n_{e}$ (positrons are colored in red); (d)-(f): accelerating field $E_{x}$; and $(\mathrm{g})-(\mathrm{i})$ : focusing field $\left(E_{y}-B_{z}\right)$ in $t=1 \mathrm{ps}$ (near the entry of the plasma region) with $\tau=0.62[(\mathrm{a}),(\mathrm{d}),(\mathrm{g})]$ and $t=7$ ps (near the exit of the plasma region) with $\tau=0.62[(\mathrm{~b})$, (e), (h)] and $\tau=-0.16$ [(c), (f), (i)].

scattering and can be injected. Other positrons will be scattered again towards subsequent bubbles, and tend to concentrate in the third donut and the third center bubbles. For negative delays, due to different wakefield structure and scattering trajectories, more positrons can be injected into the second donut bubble and center bubbles.

Injection can happen for a wide range of different laser and plasma parameters and with different delays. We take two cases with time delays of 0.62 and -0.16 as examples to describe the injection and acceleration dynamics as they allow injection into both donut and center bubbles and have relatively high positron charges. The electron density distribution, accelerating and focusing fields at time of 1 and $7 \mathrm{ps}$ with the two delays are shown in Fig. 4. At 1 ps, the positrons in the area, noted with black rectangles, will be focused during acceleration as they experience both positive $E_{x}$ and negative focusing field gradient. The focusing field can also possibly trap positrons passing through this area, which is the process of injection. The positrons can be accelerated in the bubbles after injection. At $7 \mathrm{ps}$, most of the injected positrons are accelerated to high energy (shown in Fig. 5), then most of them will start to decelerate. It is worthy to note that $98 \%$ of injected positrons can be accelerated to more than $80 \mathrm{MeV}$ both in the center and donut bubbles in the case of $\tau=0.62$, while only $37 \%$ of injected positrons can be accelerated to higher than $80 \mathrm{MeV}$ in the case of $\tau=-0.16$, and remarkably more positrons are injected in the center bubbles.

In the case of $\tau=0.62$ [shown as Fig. 5(a)], all four bubbles can generate quasimonoenergetic positron beams
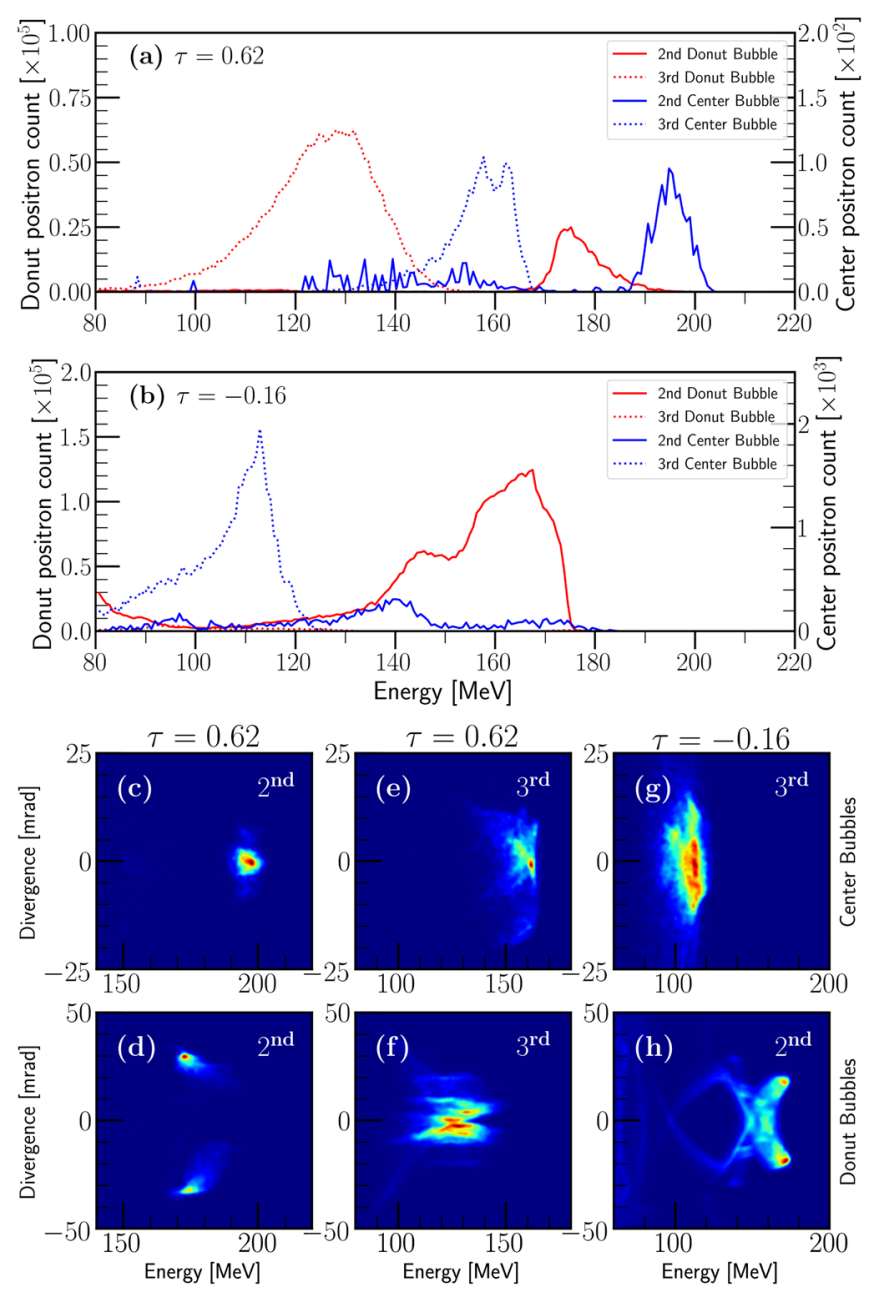

FIG. 5. (a) and (b): typical energy spectra of accelerated positrons in the second and third bubbles of (a) $\tau=0.62$ and (b) $\tau=-0.16$ at $t=7$ ps [corresponds to Figs. 4(b) and 4(c), respectively]. (c) to (h): profiles of positron beams from different bubbles: (c) $\tau=0.62$ second center bubble; (d) $\tau=0.62$ second donut bubble; (e) $\tau=0.62$ third center bubble; (f) $\tau=0.62$ third donut bubble; (g) $\tau=-0.16$ third center bubble; (h) $\tau=-0.16$ second donut bubble.

[Figs. 5(c)-5(f)]. About $99.8 \%$ of injected positrons (2.06 pC in total) are from the donut bubbles. The relative energy spread $\left(\delta_{E}=\Delta E / E_{\text {peak }}\right.$, where $\Delta E$ and $E_{\text {peak }}$ are the energy range in FWHM and the peak energy of the positron bunch) of the accelerated positrons in the second and third donut bubbles is roughly $6 \%$ and $20 \%$ at peak energies of about 170 and $120 \mathrm{MeV}$, respectively. The relative energy spread of the positrons is roughly 5\% and $10 \%$ at peak energies of about 200 and $150 \mathrm{MeV}$ in the second and third center bubbles, respectively. The center bubbles can generate low divergence and monoenergetic positron "dots," while donut bubbles generate positron "rings" with much higher charge. In the case of $\tau=-0.16$ [shown as Fig. 5(b)], the accelerated positrons concentrate in the second [Fig. 5(h)] and the third center [Fig. 5(g)] bubbles due to different scattering patterns, and the positron 
charge in center bubbles can be greatly increased compared with that in the case of $\tau=0.62$. However, they have much larger divergence angles.

The results suggest that the optimization of the relative energy spread or the total charge in the acceleration process can be done through the fine-tuning of the time delay $\tau$. In the simulation, the magnitude of the accelerating field for positrons is estimated to be the same as that for electrons. Similarly, high-energy positrons can also be achieved through increasing the acceleration distance via further optimization of the matching parameters of both laser and plasma densities as those for electrons $[9,10,12,22]$.

\section{CONCLUSION}

In conclusion, an injection method for positrons in laser wakefield acceleration is proposed by using coaxially propagating ring-shaped and Gaussian laser beams. The dynamical processes experienced by the positronsscattering, injection and acceleration-are analyzed and confirmed through PIC simulations. The simulations show that relatively high-charged, quasimonoenergetic positron beams with peak energy of around $200 \mathrm{MeV}$ can be achieved, and such high-energy collimated positron beams are appropriate for applications and further experiments.

\section{ACKNOWLEDGMENTS}

The work has been supported by the National Key Research and Development Program of China (Grants No. 2016YFA0401100 and No. SQ2016zy04003194), the NSFC (Grant No. 11575011), and the Science Challenging Project (Grant No. TZ2017005). Simulations were carried out at High Performance Computing Platform in Peking University.

[1] C. M. Surko and R. G. Greaves, Emerging science and technology of antimatter plasmas and trap-based beams, Phys. Plasmas 11, 2333 (2004).

[2] L. Accardo et al., High Statistics Measurement of the Positron Fraction in Primary Cosmic Rays of 0.5-500 GeV with the Alpha Magnetic Spectrometer on the International Space Station, Phys. Rev. Lett. 113, 121101 (2014).

[3] J. F. C. Wardle, D. C. Homan, R. Ojha, and D. H. Roberts, Electron-positron jets associated with the quasar 3C279, Nature (London) 395, 457 (1998).

[4] G. Weidenspointner, G. Skinner, P. Jean, J. Knodlseder, P. von Ballmoos, G. Bignami, R. Diehl, A. W. Strong, B. Cordier, S. Schanne, and C. Winkler, An asymmetric distribution of positrons in the Galactic disk revealed by gamma-rays, Nature (London) 451, 159 (2008).

[5] T. Tajima and J. M. Dawson, Laser Electron Accelerator, Phys. Rev. Lett. 43, 267 (1979).

[6] J. Faure, Y. Glinec, A. Pukhov, S. Kiselev, S. Gordienko, E. Lefebvre, J.-P. Rousseau, F. Burgy, and V. Malka,
A laser-plasma accelerator producing monoenergetic electron beams, Nature (London) 431, 541 (2004).

[7] C. G. R. Geddes, C. Toth, J. van Tilborg, E. Esarey, C. B. Schroeder, D. Bruhwiler, C. Nieter, J. Cary, and W. P. Leemans, High-quality electron beams from a laser wakefield accelerator using plasma-channel guiding, Nature (London) 431, 538 (2004).

[8] S. P. D. Mangles, C. D. Murphy, Z. Najmudin, A. G. R. Thomas, J. L. Collier, A. E. Dangor, E. J. Divall, P. S. Foster, J. G. Gallacher, C. J. Hooker et al., Monoenergetic beams of relativistic electrons from intense laser-plasma interactions, Nature (London) 431, 535 (2004).

[9] W. Lu, C. Huang, M. Zhou, W. B. Mori, and T. Katsouleas, Nonlinear Theory for Relativistic Plasma Wakefields in the Blowout Regime, Phys. Rev. Lett. 96, 165002 (2006).

[10] J. S. Liu, C. Q. Xia, W. T. Wang, H. Y. Lu, C. Wang, A. H. Deng, W. T. Li, H. Zhang, X. Y. Liang, Y. X. Leng et al., All-Optical Cascaded Laser Wakefield Accelerator Using Ionization-Induced Injection, Phys. Rev. Lett. 107, 035001 (2011).

[11] X. M. Wang, R. Zgadzaj, N. Fazel, Z. Y. Li, S. A. Yi, X. Zhang, W. Henderson, Y. Y. Chang, R. Korzekwa, H. E. Tsai et al., Quasi-monoenergetic laser-plasma acceleration of electrons to $2 \mathrm{GeV}$, Nat. Commun. 4, 1988 (2013).

[12] W. P. Leemans, A. J. Gonsalves, H. S. Mao, K. Nakamura, C. Benedetti, C. B. Schroeder, C. Toth, J. Daniels, D. E. Mittelberger, S.S. Bulanov et al., Multi-GeV Electron Beams from Capillary-Discharge-Guided Subpetawatt Laser Pulses in the Self-Trapping Regime, Phys. Rev. Lett. 113, 245002 (2014).

[13] W. P. Leemans, B. Nagler, A. J. Gonsalves, C. Tóth, K. Nakamura, C. G. R. Geddes, E. Esarey, C. B. Schroeder, and S. M. Hooker, GeV electron beams from a centimetrescale accelerator, Nat. Phys. 2, 696 (2006).

[14] H. Lu, M. Liu, W. Wang, C. Wang, J. Liu, A. Deng, J. Xu, C. Xia, W. Li, H. Zhang et al., Laser wakefield acceleration of electron beams beyond $1 \mathrm{GeV}$ from an ablative capillary discharge waveguide, Appl. Phys. Lett. 99, 091502 (2011).

[15] J. Faure, C. Rechatin, A. Norlin, A. Lifschitz, Y. Glinec, and V. Malka, Controlled injection and acceleration of electrons in plasma wakefields by colliding laser pulses, Nature (London) 444, 737 (2006).

[16] I. Blumenfeld, C. E. Clayton, F. J. Decker, M. J. Hogan, C. K. Huang, R. Ischebeck, R. Iverson, C. Joshi, T. Katsouleas, N. Kirby et al., Energy doubling of $42 \mathrm{GeV}$ electrons in a metrescale plasma wakefield accelerator, Nature (London) $\mathbf{4 4 5}$, 741 (2007).

[17] W. Lu, M. Tzoufras, C. Joshi, F. S. Tsung, W. B. Mori, J. Vieira, R. A. Fonseca, and L. O. Silva, Generating multi$\mathrm{GeV}$ electron bunches using single stage laser wakefield acceleration in a 3D nonlinear regime, Phys. Rev. ST Accel. Beams 10, 061301 (2007).

[18] S. Kneip, S. R. Nagel, S. F. Martins, S. P. D. Mangles, C. Bellei, O. Chekhlov, R. J. Clarke, N. Delerue, E. J. Divall, G. Doucas et al., Near-GeV Acceleration of Electrons by a Nonlinear Plasma Wave Driven by a Self-Guided Laser Pulse, Phys. Rev. Lett. 103, 035002 (2009).

[19] T. Kameshima, W. Hong, K. Sugiyama, X. Wen, Y. Wu, C. Tang, Q. Zhu, Y. Gu, B. Zhang, H. Peng et al., $0.56 \mathrm{GeV}$ 
laser electron acceleration in ablative-capillary-discharge plasma channel, Appl. Phys. Express 1, 066001 (2008).

[20] C. E. Clayton, J. E. Ralph, F. Albert, R. A. Fonseca, S. H. Glenzer, C. Joshi, W. Lu, K. A. Marsh, S. F. Martins, W. B. Mori et al., Self-Guided Laser Wakefield Acceleration Beyond $1 \mathrm{GeV}$ Using Ionization-Induced Injection, Phys. Rev. Lett. 105, 105003 (2010).

[21] S. Steinke, J. van Tilborg, C. Benedetti, C. G. Geddes, C. B. Schroeder, J. Daniels, K. K. Swanson, A. J. Gonsalves, K. Nakamura, N. H. Matlis et al., Multistage coupling of independent laser-plasma accelerators, Nature (London) 530, 190 (2016).

[22] A. J. Gonsalves, K. Nakamura, J. Daniels, C. Benedetti, C. Pieronek, T. C. H. de Raadt, S. Steinke, J. H. Bin, S. S. Bulanov, J. van Tilborg et al., Petawatt Laser Guiding and Electron Beam Acceleration to $8 \mathrm{GeV}$ in a Laser-Heated Capillary Discharge Waveguide, Phys. Rev. Lett. 122, 084801 (2019).

[23] G. Sarri, W. Schumaker, A. Di Piazza, M. Vargas, B. Dromey, M. E. Dieckmann, V. Chvykov, A. Maksimchuk, V. Yanovsky, Z. H. He et al., Table-Top Laser-Based Source of Femtosecond, Collimated, Ultrarelativistic Positron Beams, Phys. Rev. Lett. 110, 255002 (2013).

[24] H. Chen, S. C. Wilks, J. D. Bonlie, E. P. Liang, J. Myatt, D. F. Price, D. D. Meyerhofer, and P. Beiersdorfer, Scaling the Yield of Laser-Driven Electron-Positron Jets to Laboratory Astrophysical Applications, Phys. Rev. Lett. 102, 105001 (2009).

[25] H. Chen, S. C. Wilks, D. D. Meyerhofer, J. Bonlie, C. D. Chen, S. N. Chen, C. Courtois, L. Elberson, G. Gregori, W. Kruer et al., Relativistic Positron Creation Using Ultraintense Short Pulse LasersPhys. Rev. Lett. 105, 015003 (2010).

[26] H. Chen, F. Fiuza, A. Link, A. Hazi, M. Hill, D. Hoarty, S. James, S. Kerr, D. D. Meyerhofer, J. Myatt et al., Relativistic Quasimonoenergetic Positron Jets from Intense Laser-Solid Interactions, Phys. Rev. Lett. 114, 215001 (2015).

[27] A. S. Firouzjaei and B. Shokri, Trapping and acceleration of hollow electron and positron bunch in a quasi-linear donut wakefield, Phys. Plasmas 24, 013107 (2017).
[28] L. L. Yu, C. B. Schroeder, F. Y. Li, C. Benedetti, M. Chen, S. M. Weng, Z. M. Sheng, and E. Esarey, Control of focusing fields for positron acceleration in nonlinear plasma wakes using multiple laser modes, Phys. Plasmas 21, 120702 (2014).

[29] J. Vieira and J. T. Mendonca, Nonlinear Laser Driven Donut Wakefields for Positron and Electron Acceleration, Phys. Rev. Lett. 112, 215001 (2014).

[30] S. Gessner, E. Adli, J. M. Allen, W. An, C. I. Clarke, C. E. Clayton, S. Corde, J. P. Delahaye, J. Frederico, S. Z. Green et al., Demonstration of a positron beam-driven hollow channel plasma wakefield accelerator, Nat. Commun. 7, 11785 (2016).

[31] C. B. Schroeder, C. Benedetti, E. Esarey, and W. P. Leemans, Beam loading in a laser-plasma accelerator using a nearhollow plasma channel, Phys. Plasmas 20, 123115 (2013).

[32] T. Esirkepov, S. V. Bulanov, M. Yamagiwa, and T. Tajima, Electron, Positron, and Photon Wakefield Acceleration: Trapping, Wake Overtaking, and Ponderomotive Acceleration, Phys. Rev. Lett. 96, 014803 (2006).

[33] W. Lu, C. Huang, M. M. Zhou, W. B. Mori, and T. Katsouleas, Limits of linear plasma wakefield theory for electron or positron beams, Phys. Plasmas 12, 063101 (2005).

[34] I. Kostyukov, A. Pukhov, and S. Kiselev, Phenomenological theory of laser-plasma interaction in "bubble" regime, Phys. Plasmas 11, 5256 (2004).

[35] T. D. Arber, K. Bennett, C.S. Brady, A. LawrenceDouglas, M. G. Ramsay, N. J. Sircombe, P. Gillies, R. G. Evans, H. Schmitz, A. R. Bell, and C. P. Ridgers, Contemporary particle-in-cell approach to laser-plasma modelling, Plasma Phys. Controlled Fusion 57, 113001 (2015).

[36] See Supplemental Material at http://link.aps.org/ supplemental/10.1103/PhysRevAccelBeams.23.091301 for the detailed simulation setups, how the charge and spatial distribution of positrons are estimated from 2D simulations, and how 2D simulations are validated against 3D simulations. It also describes how to prepare the required laser beams and positrons for possible experiments. 